\title{
Current Relative Value Unit Scale Does Not Appropriately Compensate for Longer Orthopedic Sports Surgeries
}

\author{
Trevor Simcox, M.D., Jason Kreinces, B.S., Daniel Tarazona, M.D., Ioannis Zouzias, M.D., \\ and Mark Grossman, M.D.
}

\begin{abstract}
Purpose: To assess whether reimbursement for orthopaedic sports procedures adequately compensates for operative time and surgical complexity. Methods: The National Surgical Quality Improvement Program (NSQIP) database was queried for all orthopedic sports medicine procedures performed greater than 150 times from 2016 to 2018 with regard to operative time, preoperative risk factors, morbidity, and mortality data. Physician work relative value units (wRVU) data were obtained from the 2020 Centers for Medicare \& Medicaid Services (CMS) fee schedule. The primary outcome was wRVU per minute operative time (wRVU/min). Linear regressions were used to assess wRVU, operative time, and wRVU/ min. Results: A total of 42 CPT codes, including 84,966 cases, were stratified into the top and bottom $50 \%$, according to mean operative time, complications, mortality, reoperations, and readmissions. Mean wRVU/min was significantly lower for longer procedures (.153 vs .187; $P=.02)$, and comparable with regard to ASA score, complications, mortality, readmissions, and reoperations. Arthroscopy reimbursed more (.187 vs .148 wRVU/min; $P=.008)$, with lower complications ( 1.5 vs $2.6 \% ; P=.115$ ) and operative time (56.1 vs $82.8 \mathrm{~min} ; P=.001$ ) compared to open. Multivariate linear regression revealed that after adjusting for complication rate, there was a decrease of $.054 \mathrm{wRVU} / \mathrm{h}(P=.026)$ and $\$ 116.90 /$ hour less for every additional hour of operative time. Conclusion: The current 2020 RVU scale does not fairly compensate sports procedures with longer operative times. When examining the hourly reimbursement rates for the most commonly performed sports procedures, there is a significant trend toward lower reimbursement for longer procedures even after accounting for complication rates. Furthermore, procedures of the knee reimbursed at higher rates relative to the general pool of sports procedures and open procedures are compensated at a lower rate compared to arthroscopic procedures.
\end{abstract}

\section{Introduction}

$\mathbf{H}$ ealth care costs in the United States are the highest per capita in the world, and health care spending is expected to far outpace both inflation and total growth of the U.S. economy for the next several years. ${ }^{1,2}$ Orthopedic surgeons, among other health care providers, should expect great change over the coming

From the Department of Orthopedic Surgery, New York University Langone Winthrop Hospital, New York University Langone Health, Mineola, New York, U.S.A. (T.S., D.T., I.Z., M.G.); and New York Medical College, Valhalla, New York, U.S.A. (J.K).

Received April 26, 2021; accepted September 11, 2021.

Address correspondence to Trevor Simcox, M.D., Department of Orthopedic Surgery NYU Langone Winthrop Hospital, NYU Langone Health, 259 1st St., Mineola, NY 11501,U.S.A.E-mail:Trevor.Simcox@nyulangone.org

(C) 2021 THE AUTHORS. Published by Elsevier Inc. on behalf of the Arthroscopy Association of North America. This is an open access article under the CC BY-NC-ND license (http://creativecommons.org/licenses/by-nc-nd/4.0/).

2666-061X/21590

https://doi.org/10.1016/j.asmr.2021.09.009 decades regarding reimbursement to control costs at both the institutional and federal level. It is important for orthopedists to understand not only the trends in reimbursement over time, but also the current reimbursement trends across the breadth of procedures an orthopedist performs. A comprehensive understanding of current reimbursement trends is integral for the financial success of any orthopedic practice. ${ }^{2}$

The most common method for measuring physician productivity is the work relative value units (wRVU), which is used by Centers of Medicare $\&$ Medicaid Services (CMS) to determine reimbursement payments. wRVUs are also used for physician reimbursement in hospital-based orthopedic practices. Physician reimbursements are directly tied to wRVU, and so it is imperative that the number of wRVUs assigned to a given procedure accurately reflect the work performed.

The number of wRVUs assigned to a procedure is based on operative time (including preoperative planning), mental effort, skill to complete procedure, and 
physician stress associated with performing the procedure. ${ }^{3,4}$ There has been increasing interest by the medical community in the overarching trends in the current wRVU scale and several recent studies have found a mismatch between the work performed for a procedure and the reimbursement rate..$^{5-8}$ Prior studies in the orthopedic literature have compared reimbursement for specific Current Procedural Terminology (CPT) codes and found that typically longer and more complex procedures are not appropriately compensated when compared to shorter ones. ${ }^{9-14}$

Understanding reimbursement patterns is the first step in identifying any mismatches between reimbursement and work performed for categories of procedures to help direct policy changes by subspecialty organizations. Understanding these patterns also better enables orthopedists to advocate for fair compensation for a given procedure. To date, there is a paucity of orthopedic literature that examines global trends in reimbursement for sports procedures. The purpose of this study was to assess whether reimbursement for orthopaedic sports procedures adequately compensates for operative time and surgical complexity. We hypothesized that the 2020 wRVU scale does not adequately compensate orthopedists for sports procedures that have longer operative times.

\section{Materials and Methods}

\section{Database}

The American College of Surgeons National Surgical Quality Improvement Program (NSQIP) database was reviewed from 2016 to 2018 for demographic characteristics, patient comorbidity data, operative time, and morbidity and mortality data. The NSQIP database enables surgeons to access highly reliable data on operative time and postoperative complications for clinical analysis. This data is collected from patients undergoing surgery at one of approximately 700 participating hospitals in the United States. ${ }^{15}$

\section{Study Population}

The database was queried for all cases coded under the orthopedics subspecialty and CPT codes were manually reviewed by two orthopedic surgery residents, as to if it constitutes a sports medicine procedure. Accreditation Council for Graduate Medical Education (ACGME) case log guidelines for orthopedic sports medicine fellowships were used as a reference to determine which CPT codes fall within the scope of practice of a sports surgeon. Also, CPT codes related to shoulder arthroplasty and open tendon reconstruction were also categorized as sports medicine procedures because many sports medicine physicians commonly perform these procedures and sports medicine fellowships offer shoulder arthroplasty training. Cases missing
CPT codes, wRVU, and operative time were excluded. Procedures that reimbursed less than one wRVU, or surgical procedures that had an operative time greater than 600 minutes or less than 10 minutes were excluded, as these cases do not typically represent a major orthopedic sports procedure.

Procedures performed equal to or more than 150 times during 2016-2018 were included. Meanwhile, procedures performed less than 50 times per year on average, as determined by the database, were excluded because these are uncommonly performed by generally practicing sports surgeons. Cases with multiple coded CPTs (greater than 1 CPT code) were excluded because secondary wRVUs are typically not reimbursed at the full rate and the total wRVUs assigned to these cases are not representative of the actual reimbursement rate. Furthermore, it appears that the order of coding a given series of CPTs is not consistent between surgeons within the NSQIP database.

\section{Variables Studied}

The primary outcome variable considered in the study was wRVU/min. This was calculated by dividing wRVU obtained from the 2020 CMS fee schedule, by mean operative time for each CPT code, which was obtained from the NSQIP database. Operative time does not include anesthesia or perioperative time and is recorded in minutes. Reimbursement rate is also reported in units of wRVU per hour (wRVU/hour), as this is more applicable for understanding reimbursement implications. This was calculated by dividing the number of wRVUs assigned to a CPT by the mean operative time for that CPT. Secondary outcome variables studied for each CPT code were 30-day major complication rate, readmission, reoperation, and American Society of Anesthesiologists (ASA) score. Deep organ infection, deep wound infection, surgical site dehiscence, renal failure, pulmonary embolism or deep vein thrombosis, cerebrovascular accident, myocardial infarction, cardiac arrest, bleeding requiring transfusion, sepsis, postoperative ventilator dependence, unexpected reintubation, and death are the major complications that were considered in the study. One assumption of this study is that the overall complication rate is likely to be related to physician stress, mental effort, and surgical risk, such that postoperative major complication rate should theoretically correlate with the reimbursement for a given procedure. ${ }^{16-18}$

\section{Statistical Analysis}

Univariate linear regression and multiple regression were used to model the association between wRVU, wRVU/hour, operative time, and complication rate. CPTs were stratified into two cohorts based on mean operative time, representing the longest $50 \%$ and shortest $50 \%$ procedures. CPTs were also stratified into 
Fig 1. Scatterplot of wRVU and mean operative time. Blue dots represent individual $\mathrm{CPT}$ codes. Univariate linear regression (red line) is .147, SE: $0.018, P<.001$, $R^{2}=.61$.

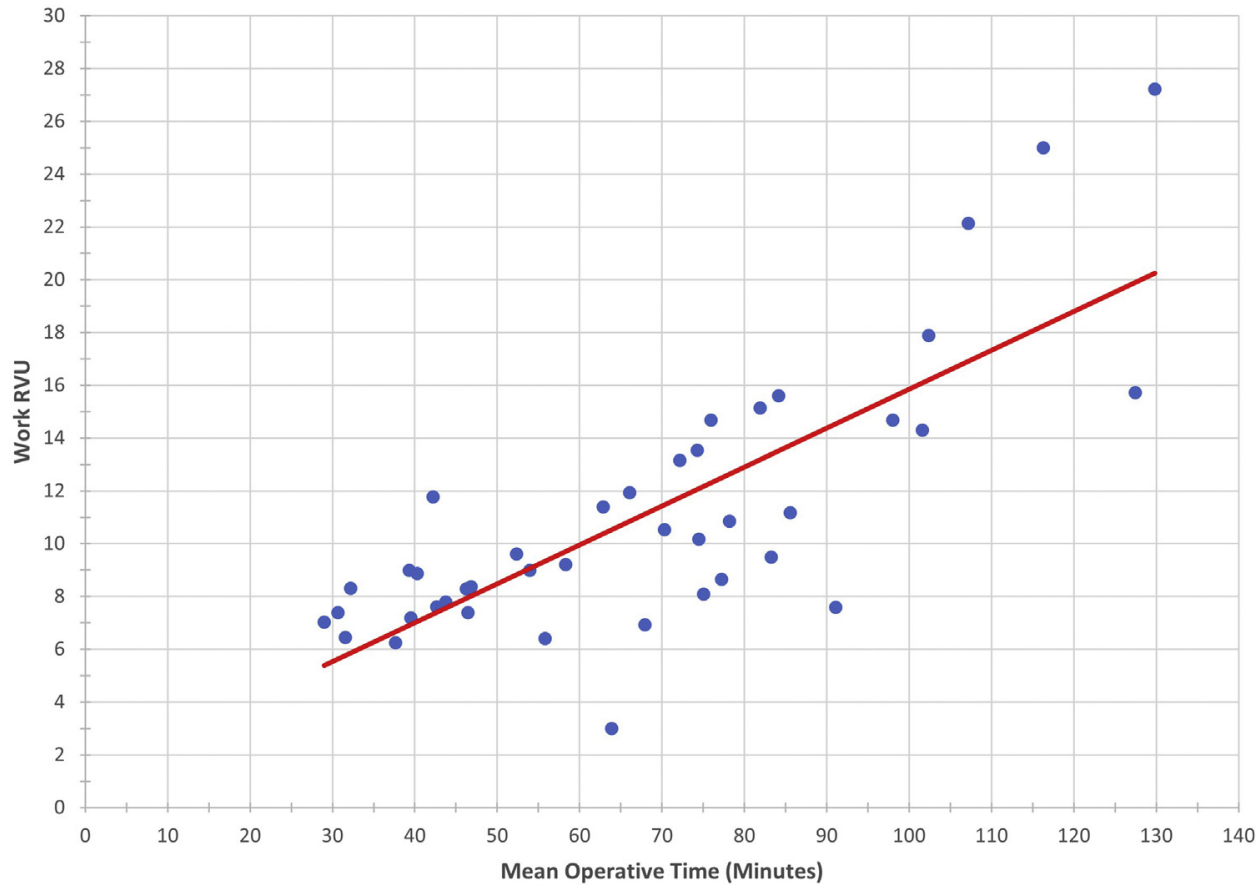

the highest and lowest $50 \%$ in terms of major complication rate, mortality rate, ASA class, reoperation rate, and readmission rate. CPTs were compared regarding anatomic location of the procedure and if arthroscopy was used. Student $t$-tests or Wilcoxon tests were used to compare wRVU/min, complication rate, and operative time for each of the CPT cohorts. Analysis of covariance was used to compare mean $\mathrm{wRVU} / \mathrm{min}$ between CPT cohorts, controlling for mean complication rate. $P$ values $<.05$ were considered statistically significant.

\section{Results}

A total of 617,676 orthopedic surgeries were performed between the years 2016 and 2018 that had complete CPT coding data. 27,645 cases were excluded because they were performed less than 50 times per year, 483,035 cases were excluded because they did not pertain to the field of sports medicine, and finally 22,000 cases were excluded due to having more than 1 CPT coded for the procedure. A total of 42 CPT codes remained and met inclusion criteria for the study, encompassing 84,996 surgical procedures. The mean wRVU was $11.06 \pm 4.96$, operative time was $65.0 \pm$ 26.4 minutes, wRVU/min was .170 \pm .047 , and major complication rate was $1.95 \pm 1.77 \%$. The 3 most poorly reimbursed procedures in terms of $\mathrm{wRVU} / \mathrm{min}$ (CPT code, wRVU/min) were arthroscopic subacromial decompression (29826, .047), open reduction internal fixation of an acromioclavicular dislocation (23550, .083 ), and quadriceps or hamstring muscle repair
$(27385, .102)$. On the other hand, the three procedures with highest reimbursement were arthroscopic medial and lateral meniscus repair $(29883, .279)$, arthroscopic chondroplasty of knee $(29877, .258)$, and arthroscopic medial or lateral partial meniscectomy $(29881, .242)$. The complete list of procedures studied with reimbursement rate may be found in the supplemental table in the appendix (Supplemental Table S1).

There was a positive correlation between wRVU and mean operative time $(.147,95 \%$ CI: .110, .184, $P<$ $.001, R^{2}=.61$, Fig 1). When comparing mean reimbursement rate to operative time and to complication rate, there was a decrease in reimbursement of .0006 $\mathrm{wRVU} / \mathrm{min}$ or $.037 \mathrm{wRVU} /$ hour for every additional minute of mean operative time and an increase of .302 $\mathrm{wRVU} /$ hour for every additional increase of $1 \%$ of mean complication rate (Fig 2, Table 1). When we use the 2020 Medicare reimbursement rate of $\$ 36.09$ per wRVU, this equates to a decrease of $\$ 80.12$ /hour (or $2.22 \mathrm{wRVU} /$ hour) for every additional hour of mean operative time and an increase in $\$ 10.90 /$ hour for every additional percent of mean complication rate. Our multivariate regression model found that after controlling for complication rate, the adjusted decrease in reimbursement was .054 wRVU/hour for every additional minute of mean operative time, equating to a loss of $\$ 116.90 /$ hour for every additional hour of mean operative time.

By stratifying CPT codes into cohorts, procedures in the top half with regard to operative time had a 

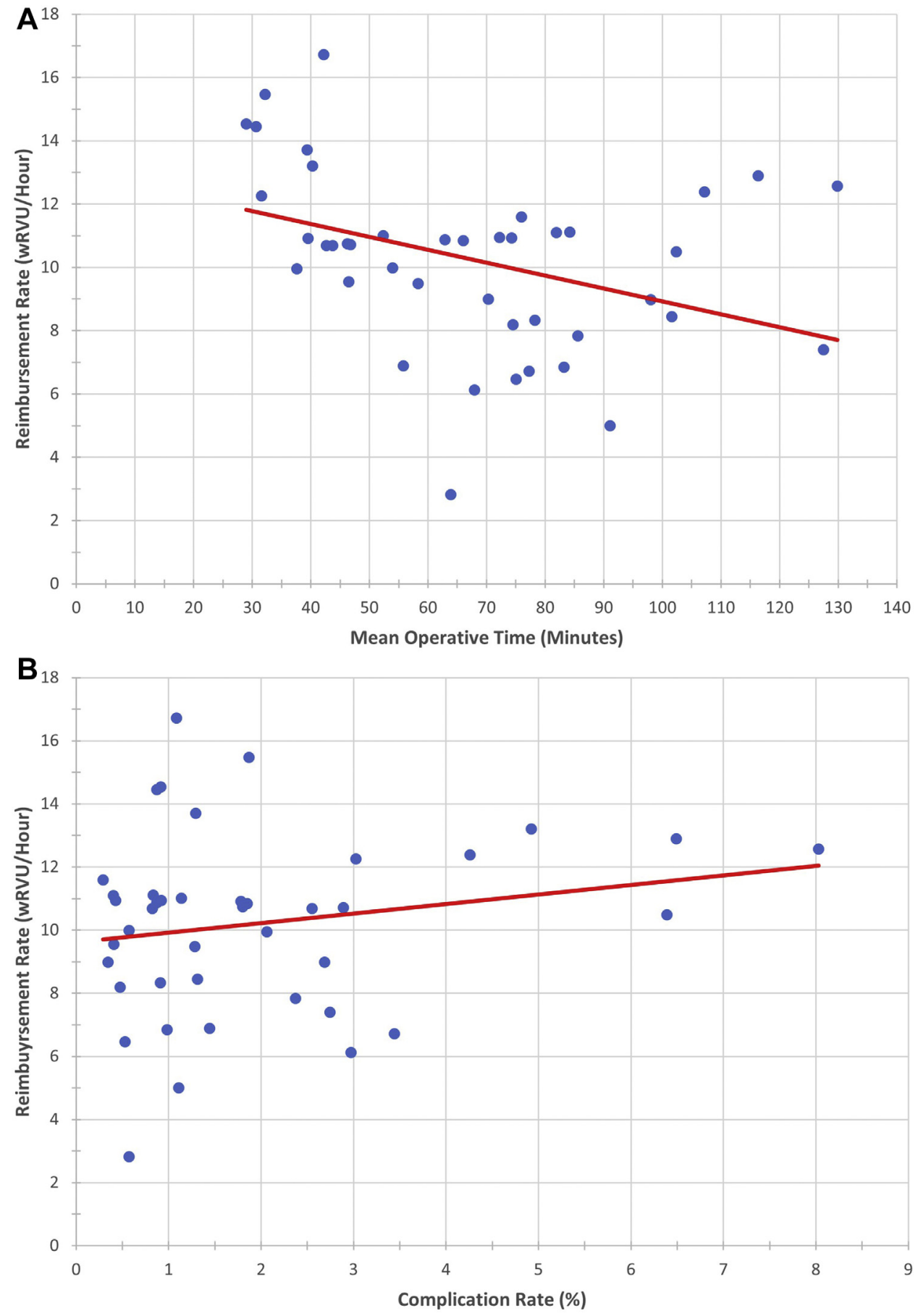

Fig 2. Scatterplots of wRVU/hour and mean operative time or complication rate with blue dots representing individual $\mathrm{CPT}$ codes. (A) Univariate linear regression depicting mean operative and wRVU/hour (red line) is -0.037 , SE: $.016, P=.026, R^{2}=$ .344. (B) Univariate linear regression depicting complication rate and wRVU/hour (red line) is .629 , SE: $.242, P=.013, R^{2}=$ 0.499 .

Table 1. Multiple Regression of Reimbursement Rate (wRVU/hour), Mean Operative Time, and Mean Complication Rate

\begin{tabular}{lccccccc}
\hline & \multicolumn{3}{c}{ Unadjusted Model } & & \multicolumn{3}{c}{ Adjusted Model } \\
\cline { 2 - 3 } \cline { 5 - 7 } & Coefficient & R-Squared & $P$ Value & & Coefficient (Standard Error) & $R$-Squared & $P$ Value \\
\hline Mean operative time (minutes) & $-.037(.016)$ & .119 & $.026^{*}$ & & $-054(0.16)$ & .211 & $.002^{*}$ \\
Complication rate $(\%)$ & $.302(.247)$ & .036 & .229 & & $.629(0.242)$ & $.013^{*}$ \\
\hline
\end{tabular}

$$
{ }^{*} P<.05
$$


Table 2. Univariate Analysis of wRVU/min

\begin{tabular}{|c|c|c|c|}
\hline & No. CPTs & Mean wRVU/min & $P$ Value \\
\hline \multicolumn{4}{|c|}{ Operative time (Minutes) } \\
\hline Less than 67 & 21 & $0.187 \pm 0.050$ & $0.020^{*}$ \\
\hline 67 or more & 21 & $0.153 \pm 0.039$ & \\
\hline \multicolumn{4}{|l|}{ Major } \\
\hline \multicolumn{4}{|c|}{ complication rate $(\%)$} \\
\hline Less than 1.3 & 21 & $0.166 \pm 0.053$ & 0.607 \\
\hline 1.3 or more & 21 & $0.174 \pm 0.042$ & \\
\hline \multicolumn{4}{|l|}{ Mortality rate (\%) } \\
\hline 0 & 23 & $0.164 \pm 0.044$ & 0.338 \\
\hline Above 0 & 19 & $0.178 \pm 0.051$ & \\
\hline \multicolumn{4}{|l|}{ ASA Class (score) } \\
\hline less than 2 & 21 & $0.165 \pm 0.050$ & 0.451 \\
\hline 2 or more & 21 & $0.176 \pm 0.045$ & \\
\hline \multicolumn{4}{|c|}{ Reoperation rate (\%) } \\
\hline Less than 0.6 & 21 & $0.171 \pm 0.052$ & 0.914 \\
\hline 0.6 or more & 21 & $0.169 \pm 0.044$ & \\
\hline \multicolumn{4}{|c|}{ Readmission rate (\%) } \\
\hline Less than 1.1 & 21 & $0.169 \pm 0.054$ & 0.854 \\
\hline 1.1 or greater & 21 & $0.172 \pm 0.041$ & \\
\hline
\end{tabular}

significantly lower reimbursement rate than those in the bottom half $(.153 \pm .039$ vs. $.187 \pm .050 ; P=.02$, Table 2). There were no significant differences in reimbursement between procedures in the top versus the bottom half of codes with regard to major complication rate $(.174 \pm .042$ vs $.166 \pm .053 ; P=.607$, Table 2$)$, mortality rate $(.178 \pm .051$ vs $.164 \pm .044 ; P=$ .338 , Table 2$)$, ASA score $(.176 \pm .045$ vs $.165 \pm .050$; $P=.451$, Table 2$)$, reoperation rate $(.169 \pm .044 \mathrm{vs} .171$ $\pm .052 ; P=.914$, Table 2$)$, and readmission rate $(.172 \pm$ .041 vs $.169 \pm .054 ; P=.854$, Table 2 ).

When comparing wRVU/min for procedures with regard to anatomic location, knee procedures $(.203 \pm$ .048 vs .156 $\pm .040 ; P=.002$, Table 3 ) and procedures involving arthroscopy $(.187 \pm .047 \mathrm{vs} .148 \pm .040 ; P=$ .008 , Table 3) had significantly higher reimbursement rates; this was true even after adjusting for differences in complication rate. There were no significant differences between procedures performed on upper versus lower extremity $(.161 \pm .041$ vs $.183 \pm .053 ; P=.137)$ or between procedures performed on the shoulder versus other anatomic locations $(.166 \pm .040$ vs $.174 \pm$ $.054 ; P=.619)$. Shoulder arthroplasty cases trended toward higher reimbursement but were not significantly different from the general pool of sports procedures $(.201 \pm .180$ vs .167 $\pm .049 ; P=.169)$.

Surgeries involving the knee $(46.1 \pm 20.9$ vs $\pm 77.1 \pm$ $23.4 \mathrm{~min} ; P<.001$, Table 3$)$ and arthroscopic procedures $(56.1 \pm 22.5$ vs $82.8 \pm 24.6 \mathrm{~min} ; P=.001)$ were found to have shorter mean operative times. In contrast, surgeries involving the upper extremity (79.1 \pm 24.9 vs $52.1 \pm 20.9 \mathrm{~min} ; P=.001$, Table 3 ), shoulder
$(79.1 \pm 26.7$ vs $55.9 \pm 21.6 \mathrm{~min} ; P=.004$, Table 3$)$, and shoulder arthroplasty procedures (113.9 \pm 12.1 vs 62.6 $\pm 22.8 \mathrm{~min} ; P<.001$, Table 3 ) had significantly longer mean operative times. When looking at mean complication rate, lower extremity procedures $(2.0 \pm 1.1 \%$ vs $1.9 \pm 2.2 \% ; P=.029$; Table 3 ) and shoulder arthroplasty $(6.3 \pm 1.5 \%$ vs $1.5 \pm 1.1 \% ; P=.001$, Table 3$)$ had significantly higher mean complication rates. There were no significant differences in mean complication rate in the shoulder $(2.1 \pm 2.3 \%$ vs $1.8 \pm 1.1 \% ; P=$ .134 , Table 3$)$ and knee procedures $(2.0 \pm 1.1 \%$ vs 1.9 $\pm 2.0 \% ; P=.115$, Table 3 ) and procedures that used arthroscopy $(1.5 \pm 2.3 \%$ vs $2.6 \pm 2.3 \% ; P=.115$, Table 3).

\section{Discussion}

Under the current 2020 RVU scale, sports procedures with longer mean operative times were reimbursed at a lower rate despite a strong correlation between wRVU and operative time. Arthroscopic procedures and procedures involving the knee are reimbursed at significantly higher rates than open procedures and procedures performed on other anatomic locations. Although it is generally known that some procedures reimburse more than others when factoring in operative time and case complexity, this study confirms that the number of wRVUs assigned to a particular procedure may not always correlate with realistic operative times. To the best of the authors' knowledge, there are no prior studies that examine the most frequently performed orthopedic sports CPT codes with regard to wRVU and operative time.

The process by which CMS values are given a CPT code is highly detailed and involves input from a panel of 31 physicians, called the Relative Value Scale Update Committee (RUC). Representatives from various medical and surgical subspecialties meet 3 times a year to evaluate procedures and assign them value. For each CPT code, physician survey data are collected regarding the details of a given procedure, perioperative patient care within the bundled period, and time spent performing each of these tasks, which is then used to calculate the wRVUs. There are four components to wRVUs: 1) preservice work, 2) intraservice work, 3) immediate postservice work, and 4) postoperative evaluation and management visits. ${ }^{4}$ Each component then factors in both the time it takes to perform a service and the intensity of the service. ${ }^{3}$ Although a great deal of attention is used in these calculations, the quality of the analysis is dependent on the accuracy of the data used. Prior studies have consistently demonstrated the inaccuracy of physician time estimates compared to operative records. ${ }^{19,20}$ One study has found that the mean operative times estimated by the RUC are inaccurate, resulting in inaccurate wRVU allocation. ${ }^{6}$ This study concluded that the physician 
Table 3. Univariate Analysis of Reimbursement, Complication Rate, and Operative Times by Type of Procedure

\begin{tabular}{lcccccc}
\hline \multicolumn{1}{c}{$\begin{array}{c}\text { Mean Complication } \\
\text { Comparison Groups }\end{array}$} & $\begin{array}{c}\text { Mean Operative } \\
\text { Rate }\end{array}$ & $P$ Value & $\begin{array}{c}\text { Time (minutes) } \\
\text { Up Value }\end{array}$ & $\begin{array}{c}\text { Mean RVU/min } \\
P \text { Value }\end{array} \quad P$ Value (Adjusted) \\
\hline Lower extremity & $1.9 \pm 2.2 \%$ & $.029^{*}$ & $79.1 \pm 24.9$ & $.001^{*}$ & $.161 \pm 0.041$ & .137 \\
Shoulder procedure & $2.0 \pm 1.1 \%$ & & $52.1 \pm 20.9$ & & $0.183 \pm .053$ & .145 \\
Non-shoulder procedure & $2.1 \pm 2.3 \%$ & .134 & $79.1 \pm 26.7$ & $.004^{*}$ & $.166 \pm .040$ & .619 \\
Knee procedure & $1.8 \pm 1.1 \%$ & & $55.9 \pm 21.6$ & & $.174 \pm .054$ & .565 \\
Non-knee procedure & $2.0 \pm 1.1 \%$ & .161 & $46.1 \pm 20.9$ & $<.001^{*}$ & $.203 \pm .048$ & $.002^{*}$ \\
Arthroscopic procedure & $1.9 \pm 2.0 \%$ & & $77.1 \pm 23.4$ & & $.156 \pm .040$ & $.002^{*}$ \\
Open procedure & $1.5 \pm 2.3 \%$ & .115 & $56.1 \pm 22.5$ & $.001^{*}$ & $.187 \pm .047$ & $.008^{*}$ \\
Shoulder arthroplasty & $2.6 \pm 2.3 \%$ & & $82.8 \pm 24.6$ & & $.148 \pm .040$ & $.001^{*}$ \\
Non-Shoulder arthroplasty & $6.3 \pm 1.5 \%$ & $.001^{*}$ & $113.9 \pm 12.1$ & $<.001^{*}$ & $.201 \pm .18$ & .169 \\
\hline
\end{tabular}

${ }^{*} P<.05$.

survey data and real-world operative times differ on average by $23.6 \%$ and that shorter cases generally have overestimated operative time. Another recent study showed a discrepancy between the surgeon-reported operative time to the CMS compared to operative times recorded in the ACS NSQIP database for multiple subspecialities, including orthopedics. ${ }^{21}$

For orthopedic sports procedures, we found that after adjusting for a procedure's complication rate, there was a significant decrease in the reimbursement rate equal to $3.24 \mathrm{wRVU} /$ hour for every additional hour of mean operative time. Using the 2020 Medicare wRVU reimbursement rate of $\$ 36.09$ per wRVU, this equates to a loss of $\$ 116.90 /$ hour after adjusting for complication rate. The monetary losses calculated in this study represent realistic hourly reimbursement and are more precise with the use of accurate operative times compared to those generated from physician survey data. ${ }^{21}$

When comparing the cohorts of CPT based on operative time, ASA classification, major complication, mortality, readmission, and reoperation rates, we confirmed the significant difference in mean wRVU/ min between longer and shorter procedures; furthermore, we were unable to identify significant differences in reimbursement rate for the other factors included in the cohort analysis. This is unexpected because allocation of wRVUs should also account for procedures performed on sicker patients and those with higher postoperative complication rates. This finding is consistent with one prior study of orthopedic trauma procedures showing that reimbursement is not commensurate with risk, ${ }^{9}$ although some studies from other surgical subspecialties have mixed findings regarding this correlation. ${ }^{22,23}$

When comparing procedures performed at various anatomic locations, we identified several significant trends. Primarily, knee and arthroscopic procedures were found to have significantly higher reimbursement than the general pool of orthopedic sports procedures. This is likely a result of these two categories of procedures having significantly lower mean operative time. In contrast, procedures involving the upper extremity, shoulder, and shoulder arthroplasty all had significantly higher operative times with similar wRVU/min compared to the general pool of sports procedures. Because of the ubiquitous nature of arthroscopy in contemporary sports surgery, one could argue that the pendulum has swung toward open surgical approaches having an overall higher case complexity and are more technically challenging. In the event that a skilled arthroscopist cannot achieve an acceptable result arthroscopically, the bailout is often to convert to open or mini-open techniques. The estimation of operative time by the RUC, and consequently allocation of wRVUs, still appears to favor arthroscopic procedures. Because RVU allocation exists on a relative scale, the higher reimbursement of knee and arthroscopic procedures generally comes at the expense of relatively underreimbursed sports procedures.

\section{Limitations}

There are several notable limitations to this study. In order to more accurately calculate wRVU per minute for each CPT code, procedures in which multiple CPT codes were used were deliberately excluded from this study. This may limit the generalizability of the results because a significant proportion of sports surgeries involve multiple related procedures. This analysis is not powered or designed to compare individual procedure to one another, but rather to identify global trends; we believe that the exclusion of multiplecoded procedures portrays a more accurate representation of how the RUC examines a particular CPT code. Another consideration to this study's generalizability is the fact that wRVUs may not be applicable to all practice environments, and some orthopedic practices may use alternative reimbursement structures. Furthermore, the number of wRVUs assigned to a given CPT code is not directly tied to reimbursement from private insurance companies, and there are other 
factors that influence reimbursement payments. Another possible limitation is that this study used surgical data from the years 2016-2018. We have assumed that, barring isolated examples of advancement in surgical technique, operative times and complication rates have remained more or less stable during this time period. Furthermore, the NSQIP database does not specify whether the procedure was performed by a fellowship-trained sports surgeon. It is our view that the RUC must account for generalists when calculating mean operative times; surgeons who are more efficient in the operating room and who have lower operative times are, thus, rewarded for their improved efficiency with higher hourly reimbursement rates. Lastly, on the basis of prior studies of this topic, complication rates were assumed to relate, at least somewhat, to a higher case complexity and physician stress level. ${ }^{5,22,23}$

\section{Conclusion}

The current 2020 RVU scale does not fairly compensate sports procedures with longer operative times. When examining the hourly reimbursement rates for the most commonly performed sports procedures, there is a significant trend toward lower reimbursement for longer procedures even after accounting for complication rates. Furthermore, procedures of the knee reimbursed at higher rates relative to the general pool of sports procedures and open procedures are compensated at a lower rate compared to arthroscopic procedures.

\section{References}

1. Keehan SP, Cuckler GA, Poisal JA, et al. National Health Expenditure Projections, 2019-28: Expected Rebound In Prices Drives Rising Spending Growth. Health Affairs 2020;39:704-714.

2. Eltorai AE, Durand WM, Haglin JM, et al. Trends in Medicare reimbursement for orthopedic procedures: 2000 to 2016. Orthopedics 2018;41:95-102.

3. Jacobs JP, Lahey SJ, Nichols FC, et al. How is physician work valued? Ann Thorac Surg 2017;103:373-380.

4. Wynn BO, Burgette LF, Mulcahy AW, et al. Development of a model for the validation of work relative value units for the Medicare physician fee schedule. Rand Health $Q$ 2015;5:5.

5. Chakiryan NH, Jiang DD, Gillis KA, Chen Y, Acevedo AM, Sajadi KP. Relative value units do not adequately account for operative time of urological surgery. J Urol 2020;203: 1003-1007.

6. Chakiryan NH, Jiang DD, Gillis KA, Chen Y, Acevedo AM, Sajadi KP. RUC operative time estimates are inaccurate, resulting in decreased work RVU assignments for longer urologic procedures. Urology 2020;142:94-98.

7. Acuña AJ, Jella TK, Samuel LT, Schwarzkopf R, Fehring TK, Kamath AFJJ. Inflation-adjusted Medicare reimbursement for revision hip arthroplasty: Study showing significant decrease from 2002 to 2019. J Bone Joint Surg Am 2021;103:1212-1219.

8. Simcox T, Becker J, Kreinces J, Islam S, Grossman M, Gould J. Are orthopaedic trauma surgeons adequately compensated for longer procedures? An analysis of relative value units and operative times from the ACS-NSQIP database. J Orthopaed Trauma 2020;4. e20.00163.

9. Sathiyakumar V, Thakore RV, Molina CS, Obremskey WT, Sethi MK. Does physician reimbursement correlate to risk in orthopaedic trauma? J Surg Orthopaed Adv 2017;26: 48-53.

10. Samuel LT, Grits D, Acuña AJ, Piuzzi NS, HigueraRueda CA, Kamath AF. Work relative value units do not adequately support the burden of infection management in revision knee arthroplasty. J Bone Joint Surg Am 2020;102:230-236.

11. Peterson J, Sodhi N, Khlopas A, et al. A comparison of relative value units in primary versus revision total knee arthroplasty. J Arthroplasty 2018;33:S39-S42.

12. Orr RD, Sodhi N, Dalton SE, Khlopas A, Sultan AA, Chughtai M. What provides a better value for your time? The use of relative value units to compare posterior segmental instrumentation of vertebral segments. Spine J 2018;18:1727-1732.

13. Malik AT, Scharschmidt TJ, Li M, Jain N, Khan SN. Are joint surgeons being adequately compensated for singlecomponent versus double-component revision TKA? An analysis of relative value units. J Knee Surg 2020;33: 593-596.

14. Malik AT, Khan SN, Phieffer L, Ly TV, Quatman CE. Are orthopaedic surgeons being adequately compensated for ankle fractures? An analysis of relative value units (RVUs). Foot Ankle Orthopaed 2019;4: 2473011419 S00052.

15. Shiloach M, Frencher SK Jr, Steeger JE, et al. Toward robust information: Data quality and inter-rater reliability in the American College of Surgeons National Surgical Quality Improvement Program. J Am Coll Surg 2010;210: 6-16.

16. Chrouser KL, Xu J, Hallbeck S, Weinger MB, Partin MR. The influence of stress responses on surgical performance and outcomes: Literature review and the development of the surgical stress effects (SSE) framework. Am J Surg 2018;216:573-584.

17. Aust JB, Henderson W, Khuri S, Page CP. The impact of operative complexity on patient risk factors. Annals Surg 2005;241:1024.

18. Grantcharov P, Boillat T, Elkabany S, Wac K, Rivas H. Acute mental stress and surgical performance. BJS Open 2019;3:119-125.

19. Burgette LF, Mulcahy AW, Mehrotra A, Ruder T, Wynn BO. Estimating surgical procedure times using anesthesia billing data and operating room records. Health Serv Res 2017; 52:74-92.

20. McCall N, Cromwell J, Braun P. Validation of physician survey estimates of surgical time using operating room logs. Med Care Res Rev 2006;63:764-777.

21. Uppal S, Rice LW, Spencer RJ. Discrepancies created by surgeon self-reported operative time and the effects on procedural relative value units and reimbursement. Obstet Gynecol 2021:10-1097. 
22. Shah DR, Bold RJ, Yang AD, Khatri VP, Martinez SR, Canter RJ. Relative value units poorly correlate with measures of surgical effort and complexity. J Surg Res 2014; 190:465-470.
23. Gan ZS, Wood CM, Hayon S, Deal A, Smith AB, Tan H-J, et al. Correlation of relative value units with surgical complexity and physician workload in urology. Urology 2020;139:71-77. 